INVESTIGACIÓN/RESEARCH

\title{
LAS DERIVAS DE LA COMUNICACIÓN: EL CUERPO COMO TEXTO
}

Valeria Chomnalez: Universidad Nacional del Comahue (UNCo). Argentina. valeriachomnalez@yahoo.com.ar

\section{RESUMEN}

El cuerpo se construye socialmente como objeto y espacio de comunicación. El cuerpo desacralizado de la modernidad ha cobrado una centralidad inestimable en el mundo occidental contemporáneo como escenario de construcción del sujeto, naturalizando operaciones y prácticas de modificación sobre su materialidad, desplazando su estatus de sagrado por el de objeto perfectible. Múltiples prácticas contemporáneas muestran que intervenciones corporales de diversa índole ganan presencia al tiempo que van tomando nuevas formas atravesadas por procesos culturales que involucran varios aspectos como el predominio tecnológico y el avance de lo espectacular en la vida cotidiana. El presente artículo propone explorar algunos sentidos y procesos en torno de las modificaciones corporales teniendo como horizonte, en una época definida por la fluidez, la presentación por parte de la empresa finlandesa de telecomunicaciones Nokia, de un sistema (en etapa experimental) de tatuajes vibrantes que se sincronizarían con los teléfonos inteligentes o smartphones. Sería un modo de reactualizarse en el horizonte de sentidos la idea del cuerpo híbrido, la cuestión de la trascendencia de la naturaleza humana hacia un cuerpo tecnológicamente perfeccionado que enlaza con el imperativo de conectividad y con la superación de las limitaciones del cascarón humano, corruptible y obsoleto. Esta reflexión se desprende de un trabajo etnográfico más amplio que se desarrolla con jóvenes tatuadores en la capital de la provincia de Neuquén, Argentina.

PALABRAS CLAVE: Cuerpo - Tatuaje - Espectacularización de la vida - Tecnología.

\footnotetext{
${ }^{1}$ Autora correspondiente:

Valeria Chomnalez: Becaria doctoral del Consejo Nacional de Investigaciones Científicas y Técnicas, Universidad Nacional del Comahue. Argentina.

Correo: valeriachomnalez@yahoo.com.ar
} 


\title{
COMMUNICATION'S DRIFTS: THE BODY AS TEXT
}

\begin{abstract}
The body is socially constructed as object and space of communication. Since modernity period the body has had a central importance in the western contemporary world as scenery of construction of the subject, naturalizing operations and practices of modification on its materiality, displacing its status of sacred for that of perfectible object. Several contemporary practices show that different corporal interventions gain presence and, at the same time, they are taking new forms defined by cultural processes that involve various aspects as the technological predominance and the advance of spectacle in daily life. The proposal of this article is to explore some senses and processes around the corporal modifications having as horizon in a time defined by the fluency, the patent that took out the company of telecommunications Nokia, of a system (in experimental stage) of vibrate tattoos that would synchronize with smartphones. It would appear in the horizon of senses the idea of the hybrid body, the transcendency of the human nature towards a technologically perfected body that links with the imperative of connectivity and with the overcoming of the limits of human body, corruptible and obsolete shell. This reflection is part of an ethnographic work developed with young tattooists in the capital of Neuquén's province, in Argentina.
\end{abstract}

KEYWORDS: Human body - Tattoo - Society of the spectacle - Tecnology.

\section{INTRODUCCIÓN}

La preocupación por el cuerpo es el eje central de este texto, por el cuerpo simbólico y material, el cuerpo modificado, intervenido, el cuerpo forzado hasta sus límites, el cuerpo sometido, constreñido por diversos imperativos epocales, es decir, el cuerpo de individuos, de personas atravesadas por el consumo, el deseo, la osadía, el temor. La propuesta que nos inspira es la de pensar acerca de las formas múltiples y plurales en las que la modificación del cuerpo ha ido insertándose en la vida social urbana. En el contexto que brinda la modernidad, y especialmente el avance del proceso de secularización, el cuerpo abandona el estatus sagrado y abre espacio a la intervención sobre los cuerpos, al tiempo que cierra los cuerpos al mundo, a la naturaleza, ocultando orificios y disimulando protuberancias, para construirlo en y por su aséptica individualidad.

Si coincidimos en que las prácticas que rinden culto al cuerpo se han extendido en la actualidad, posiblemente lo hagamos también respecto de la multiplicación de discursos que presentan no obstante, ciertas tensiones con las representaciones hegemónicas ligadas al cuerpo por la vía del consumo. Partiendo de que el cuerpo como espacio de acción, representación y memoria, es el escenario privilegiado en el que la experiencia deja huella, habría dos corrientes divergentes a partir de dos 
diferentes formas de intervención: el cuerpo-evidencia, en el que la piel deliberadamente marcada daría cuenta del devenir, de la experiencia del sujeto por medio del tatuaje; y el cuerpo-velado en el que se buscaría borrar los rastros que permitirían identificar recorridos e historias (incluso genéticas) en/de los cuerpos por medio de la cirugía estética. Pero en ambas aparece coincidentemente el cuerpo como instrumento -a la vez sujeto/objeto- de la satisfacción individual de deseos.

El tatuaje en occidente fue ganando visibilidad pasada la primera mitad del siglo XX; lo que no libró empero, su camino de tensiones -microscópicas tal vez- y sinuosidades, acercando y alejando a los actores y sus prácticas de los dictados hegemónicos de la sociedad contemporánea. El tatuaje es a esta altura de la historia moderna, una práctica ampliamente extendida que se imbrica sin embargo en un contexto dominado por un tipo de pensamiento técnico orientado hacia objetivos prácticos. En este sentido encontramos que surgen formas novedosas de intervención de los cuerpos: como adelantamos, el hecho puntual que inquieta esta reflexión es el desarrollo -y posible aparición en el mercado- de tatuajes-vibrantes de la firma finlandesa de tecnología, Nokia. En estos desarrollos -aunque no están disponibles para un uso masivo aún-, encontramos modos en los que el imperativo tecnológico de la (hiper)comunicación y la conectividad se cuelan literalmente en la piel, además de ir filtrando en el horizonte próximo y cercano la idea del cuerpo híbrido.

Estas modalidades digitales cuyo carácter fluido, continuo, flexible se hace cotidiano, sigue operando sobre las nociones de tiempo y espacio, trastocando fronteras, travistiendo con su forma servicial y amigable procesos que entendemos, profundizarían los mecanismos de lo que Deleuze denominó como sociedades de control. Intentaremos bucear por los derroteros de los cuerpos intervenidos, pensando en las técnicas y en las construcciones que se asocian a ellas, sabiendo que el recorrido es siempre provisorio, y está guiado más por interrogantes que por certezas.

\section{METODOLOGÍA}

La reflexión que presentamos forma parte de un trabajo más amplio en el que se vienen abordando diferentes aspectos vinculados a las culturas juveniles locales que han adquirido gran visibilidad en la última década. El recorte inicial busca conocer diversos sentidos que se construyen en torno de la práctica del tatuaje, desde la voz de los propios jóvenes que hacen del arte en la piel una forma de vida y su principal sustento económico; por eso es que se trabaja desde el paradigma cualitativo con entrevistas en profundidad a tatuadores de entre 20 y 35 años, que tienen sus estudios de tattoo en la zona centro de la capital de Neuquén, provincia ubicada en el extremo noroeste de la región conocida como Patagonia, y que de acuerdo con el último censo nacional reúne algo más de 233 mil habitantes (INDEC, 2010).

En el presente texto revisamos analíticamente algunas ideas producto de inquietudes surgidas a partir del trabajo de campo, en vinculación con conceptos que nos ayudan a abordarlas en términos teóricos abstractos, y otros procesos que tienen lugar a nivel de las sociedades, con miras a delinear vías de comprensión de ciertos fenómenos 
contemporáneos. Siguiendo la perspectiva de Geertz, asumimos que las estructuras culturales son entendidas como sistemas organizados de símbolos significativos, con lo que la cultura "no es sólo un ornamento de la existencia humana, sino que es una condición esencial de ella" (1995: 52). Conocer estas estructuras culturales se encuentra en la base de nuestras preocupaciones, como fenómenos a hacer inteligibles en pos de la comprensión situada del mundo que habitamos.

\section{ANÁLISIS Y DISCUSIÓN}

\subsection{El cuerpo-texto: lecturas del sujeto social}

Ampliamente estudiado por las teorías de la comunicación kinésica o no verbal, el cuerpo es reconocido como fuente de mensajes, producto básicamente de los aprendizajes culturales. Aunque como sostiene Judith Butler, los cuerpos son algo construido socialmente pero sobre y en intercambio con lo natural, entendiendo a la "'naturaleza' como un conjunto de interrelaciones dinámicas" (Butler; 2002: 22). Así el cuerpo sería un texto en constante escritura y reescritura, constreñido sin embargo por una variedad de regulaciones y dispositivos; en cada modelo social ha operado históricamente una estructura de poder. De hecho, en Tecnologías del yo (1990), Foucault compendia los saberes que los hombres han desarrollado acerca de sí mismos, y que han utilizado para comprender(se) y controlar(se), a esto llamó tecnologías (Foucault; 1990: 47, 48). Las tecnologías en las sociedades de control (a diferencia de las sociedades disciplinarias) explica Papalini, operan sobre el individuo, son "pautas de domesticación" auto-administradas no sólo sobre los cuerpos sino además sobre pensamientos y prácticas. En la actualidad, las tecnologías del yo operarían -no ya como formas de vigilancia y control de los cuerpos, sino- como "modulaciones (...) flexibles, ubicuas e imperceptibles", incluso bajo dispositivos agradables (Papalini; 2007: 41-45).

Nos preguntamos a propósito, qué ocurre con los cuerpos, con los sujetos. Le Bretón ofrece un recorrido que relata al cuerpo grotesco del medioevo y del Renacimiento concebidos como unidad: cuerpo y hombre son uno y una misma cosa, integrado al cosmos y al mundo por la vía del contacto con el otro como parte de sí mismo (Le Bretón; 2002a: 31). Pero sobre esta materialidad que se construye colectivamente avanza el cuerpo moderno, individual, escindido del hombre; así los cuerpos han pasado a ser envoltorios, bocetos, ensayos siempre incompletos, provisorios, a disposición de quienes los poseen: el cuerpo es propiedad de un individuo. Es a la vez límite y facilitador, es materia prima perfectible. La seducción del despliegue frondoso de cuerpos y el colorido que aporta la multiplicidad de subjetividades permea los imaginarios sociales, en este contexto en el que las nuevas tecnologías permiten el manejo de grandes cantidades de (valiosa) información que además puede ser transmitida fácil y rápidamente; el desarrollo de materiales (superación de los existentes en la naturaleza); la modificación o creación genética, todo esto prescindiendo -como común denominador- de cuerpos que deban entrar en contacto.

En esta fase del capitalismo postindustrial, la proliferación de nuevas subjetividades estaría vinculada a lo que con atinada proyección, Guy Debord (1967) conceptualizó 
como la espectacularización de la vida cotidiana; con la pregnancia de los medios de masas, el cuerpo vino a ocupar un lugar central, cotidiano, en los discursos y las prácticas de los individuos, dando lugar a un escenario en el que las subjetividades son erigidas como "mercaderías muy especiales" en constante recambio, lo que no hace sino acelerar la espiral del consumo (Sibilia; 2005: 33) en la sociedad de masas. Pero esta espiral de consumo no ocurre fortuitamente sino que responde a "un patrón cultural de comportamiento que estructura determinado modo de relación del hombre con su entorno social" señala Schnaith, quien distingue dos modalidades de consumo: el consumo productivo que se realiza para reproducir (en sentido marxista) y cuyo resultado es un producto diferente, transformado, que se devuelve al exterior; y por otro lado el consumo ingestivo impulsado por los "mecanismos más arcaicos de la gratificación psíquica" (1990: 93-95). Así, este vínculo culturalmente aprendido del hombre con el consumo, como un patrón psíquico, denegaría al sujeto social la posibilidad de alcanzar una genuina felicidad -entiende la filósofa argentina-, dado que ante la imposibilidad de satisfacer el deseo aparece el "mecanismo de las gratificaciones sustitutivas" (Schnaith; 1990: 92-97). ${ }^{2}$

Volviendo a la cuestión de la sociedad espectacular, decimos que una característica central de su espectacularidad se manifiesta en la sucesiva extinción de imágenes, lo que pone al sentido de la vista como socio esencial, siempre atento a estímulos fugaces; los imaginarios sociales se van construyendo inmersos en un contexto en el que lo visual adquiere una preponderancia que se manifiesta a la vez en el objeto de la mirada por un lado, y por otro en el sujeto que contempla; esa relación dialéctica espectacular que se entabla, en la que uno sin el otro no tienen sentido, se construye no obstante sobre la base de una distancia prudente. El sustrato que amalgama los sentidos detrás de las miradas y los cuerpos sería el deseo, que otorgaría al sujeto deseado un poder, lo que daría como resultado una relación que no sería de por sí asimétrica, aunque sí seguiría siendo de necesariedad -no hay cuerpo deseado sin sujeto que lo desee-. El deseo operaría entonces como motor del consumo: "La ideología del consumo se lucra tanto con las aspiraciones como con las frustraciones del deseo" (Schnaith; 1990: 93).

\subsection{Cuerpos, intervención y deseo}

A partir de valores como la velocidad, la juventud, la salud, la diafanidad, los discursos hegemónicos fuertemente apoyados en las construcciones publicitarias y mass mediáticas, consagran estos preceptos por los que se busca equiparar ese cuerpo disociado de la persona a la máquina; los conocimientos técnicos ayudarían a prever y controlar la naturaleza del cuerpo humano, incluso a pesar de la esencial y evidente oposición dada por su (hasta ahora) ineludible decadencia y finitud. Algunos de los métodos mas difundidos se reflejan en la multiplicación de intervenciones como las cirugías estéticas, que ponen (senos, glúteos, pómulos, labios) o quitan (arrugas, grasa, deformidades, excesos y protuberancias), o en las técnicas y complementos destinados

\footnotetext{
2 Vanina Papalini profundiza el análisis en "Sucedáneos de felicidad. Subjetividad tardomoderna y cultura contemporánea", en María Alejandra Minelli (compiladora) Miradas. Cultura y subjetividad en la Argentina finisecular. Córdoba: Alción Editora; 2008.
} 
a conservar en condiciones óptimas la materialidad del sujeto, ya sea con ejercicios físicos, la ingesta de suplementos, o diversas terapias que irían atemperando, maquillando los límites orgánicos; sin embargo y paralelamente -cual daño colateralirían despojando a los cuerpos de sus singularidades, de las huellas con que la historia particular los marcara o la genética los dotara. Pero por otro lado estaría también acercándonos a formas más radicales en las que la modificación del cuerpo ya no se centraría únicamente en su aspecto exterior sino que se pondría en juego la esencia misma del hombre; de la inteligencia artificial a la ingeniería genética, que la "nueva tecnociencia [refiere como] el 'fin de la muerte'”' (Sibilia; 2005: 53, 54).

A propósito de estas transformaciones y de que una variedad de técnicas que apuntan a mantener la juventud se concentran como espacio privilegiado en "el lugar más humano del hombre" (Le Breton; 2010: 19) nos preguntamos: ¿qué fue de la intocable singularidad del sí mismo que aportaba el rostro único e irrepetible, al que se utiliza desde el siglo XIX para identificar y controlar la "buena ciudadanía" (Le Breton; 2010: 43)? ¿Qué hay de la idea del envejecimiento digno que aporta un rostro con huellas, y del prestigio de la vejez como sinónimo de experiencia e incluso de sabiduría? ¿Acaso estos procesos dejan entrever que el cuerpo estaría perdiendo su espesura, su grosor?

Ante todo, la juventud. Poco puede hacerse para contradecir a este valor cardinal contemporáneo. Como imagen de sí, se acondiciona al rostro -de acuerdo con las posibilidades- para que evidencie lo menos posible la tragedia que el tiempo arrastra sobre él. Sin embargo, las técnicas para disimular esas marcas podrían estar generando una zona gris, una hibridación entre el rostro juvenil y el envejecido. Casi todas las caras que han sido sometidas a la buena intención del bisturí o del botox, dan como resultado la transformación de ese rostro en otra cosa que un rostro joven. Pero en la era de la digitalización, la fotografía del rostro se reduce a un dato analógico innecesario como marca de identidad; el rostro de un sujeto, de un ciudadano, como dato de memoria fotográfica deja de ser imprescindible, en cambio el reconocimiento ejecutado a partir de tecnologías digitales recurre a los datos biométricos del sujeto o, en el caso del consumidor se lo reconoce por contraseñas, códigos e historiales virtuales. $^{3}$

Por otro lado, existen máquinas que amplían el poder muscular y otras que maximizan los aparatos sensoriales humanos con los que el hombre "puede superar las limitaciones que le impusieron su cuerpo y sus sentidos", explica Weizenbaum (1978: 27); las máquinas autónomas cambiaron radicalmente la configuración de la vida humana, desde el reloj hasta llegar a la computadora, fueron abriendo camino para depositar en dispositivos tecnológicos actividades y habilidades que antes eran potestad del hombre, su cuerpo y sus sentidos. Así, la capacidad de la memoria fue

\footnotetext{
${ }^{3}$ Como ejemplo, desde julio de 2012 Argentina emite un Pasaporte Electrónico que incorpora un chip en el que se almacenan "los datos biométricos de su titular (...) Este es un avance tecnológico y un salto de calidad y seguridad, haciendo que (...) sea un documento inviolable". El país se suma con esto al "grupo de [56] estados que se encuentran a la vanguardia" en emisión de pasaportes. http://www.mininterior.gov.ar/pasaporte/ (consultado agosto/2012)
} 
trocada por la de acumulación de información; aunque está claro que el almacenamiento de información no tiene nada que ver con el desarrollo de la memoria humana y el atesoramiento de recuerdos, experiencia que implica una jerarquización además del proceso de recordar versus olvidar-, y aun menos con la sabiduría como herramienta para la acción.

Qué pasa con el cuerpo de la sociedad espectacularizada, entonces. El sujeto del cuerpo libre sin ataduras de la modernidad se vuelve sobre sí en una búsqueda obsesiva de cuidado y belleza físicos. El cuerpo, como el lenguaje de las palabras, se hace instrumento y la técnica, el pensar técnico "se prepara para dominarlo todo". La utopía de la posmodernidad, sintetiza Schmucler, imagina una sociedad "donde no sólo Dios haya sido desechado sino en la que el propio hombre se vuelva un dato superfluo. La utopía mediática piensa un ser humano espectador de sí mismo" (Schmucler; 1997: 19). Si todo se reduce a relaciones, a intercambios de imágenes, de datos, a links que se enlazan unos con otros en una sucesión infinita y veloz, nos resultará imposible reconocer la profundidad, la interioridad de nada ni de nadie, finalmente todo será resumido al exterior, la cáscara, lo que se ve es lo que es. En este sentido, a las mencionadas tecnologías del yo que operan como "máximas sociales 'sé tu mismo', 'conócete a ti mismo', 'gobiérnate a ti mismo' y 'cuídate a ti mismo' (...) se acopla una fórmula aparentemente menos compleja y más feliz: 'muéstrate a ti mismo'" (Remondino y Chomnalez; 2009). La esfera íntima de los sujetos se abre a lo público, la vida se construye para ser mostrada de acuerdo con la imagen del sí mismo que se desea proyectar.

Decíamos, el Otro aparece como instrumento del deseo del sujeto exhibido. A diferencia de la palabra profunda que se asienta en un compromiso con el Otro, en un necesario respeto que funda la interacción en una correspondencia a la interpelación, la palabra despojada de su grosor, unívoca como el cuerpo espectacular, están centrados en sí mismos sobre la base de intercambios teleológicos.

\subsection{Cuerpos tatuados, ¿cuerpos en ruptura?}

El título del presente apartado evoca la publicación de la Revista Actuel Marx (2007) "Cuerpos dominados, cuerpos en ruptura", cuyos artículos buscan dar visibilidad no sólo a cuerpos sufrientes, constreñidos por el sistema hegemónico convertidos en mercancías sino, especialmente, a cuerpos que se oponen. La pregunta que nos hacemos al incursionar en el caudaloso mundo de tinta en la piel es si en esta marcación deliberada de los cuerpos puede residir una potencia emancipadora. Antes de continuar, nos resulta pertinente recordar que al abordar esta cuestión se encuentran en nuestro horizonte como grupo sobre el que asentamos nuestras reflexiones, personas que se dedican a tatuar profesionalmente, y no todo aquel o aquella que tiene un tatuaje.

Entonces, este particular modo de marcar la piel, cuyos últimos rastros descubiertos se remontan a mas de cinco mil años, encuentra su exponente mas antiguo en la momia llamada Oetzi, hallada en los Alpes italianos; sería el cuerpo de un cazador del 
neolítico, quien tenía su espalda y rodillas tatuadas (Ganter; 2006). Sin embargo, a lo largo de la historia esta práctica fue alternativamente fomentada y prohibida, relegada como marca deshonrosa para esclavos o presidiarios, asociada a rituales curativos, entre otras; lo cierto es que muy dudosamente el tatuaje haya podido actualmente quitarse totalmente el mote de práctica salvaje. De hecho, no podemos obviar que los discursos modernos sobre la materia han procedido del ámbito médico y psicológico; los estudios vinculados han intentado establecer patrones patológicos asociados a la práctica, del sadomasoquismo al narcisismo (Reisfeld; 2005).

En la actualidad, el tatuaje no responde a una práctica ritual institucionalizada, ni se presenta como signo de jerarquía y distinción social, ni es excluyente de hombres, mujeres, adultos/as, maduros/as, insanos/as. Desde nuestra perspectiva, se destaca por ser una práctica permanente en una época de fluidez que además, no estaría legitimada en el sentido de la belleza hegemónica orientada hacia la búsqueda de algún tipo de perfección del cuerpo liso, de belleza plastificada. No obstante, se acerca al cause hegemónico si lo pensamos como una técnica de construcción del sí mismo espectacular; este imperativo eminentemente visual de la sociedad se plasma también en el cuerpo tatuado. Con todo, la relevancia de éstos reside en su materialidad, el cuerpo es el soporte del texto que dice al hombre, que da a su piel una espesura y un sentido particulares. Frente al avance algo silencioso pero sostenido de las biotecnologías por un lado, y rimbombante de la virtualidad por otro, la difusión de la práctica del tatuaje rescataría al cuerpo en y por su misma materialidad, divergiendo de las prácticas que avanzan en el sentido de sustraerle al cuerpo su función y capacidad no sólo orgánico-sensorial sino comunicativo-expresiva; el tatuaje podría ser una práctica que se lo restituyera contra el imperio de lo inmaterial y de lo efímero.

El paso del tiempo, factor central para el pensamiento dominante que erige a lo joven y bello como un valor cardinal, estaría atenuado en la práctica del tattoo dado que su relevancia no se manifestaría en la obsesiva conservación y cuidado exterior del cuerpo diáfano; de hecho encontramos que en general, el tatuaje funciona mojonando en la piel hechos, acontecimientos, incorporando a otros seres -ya sea por la inscripción de un nombre o de retratos- de la esfera privada del sujeto, que lejos de borrar o disimular el pasado lo marcan como huellas reactualizándolo cotidianamente. El sujeto tatuado podría ser considerado un documento icónico de sí mismo, de su identidad y de su historia. No exentos de arrepentimiento, en algunos casos, marcas antiguas intentan ser ocultadas bajo capas de tinta que oscurecen por completo ciertas zonas, pero incluso en estos casos ese pasado oculto resurge porque está allí la espesura oscura como evidencia. El cuerpo-texto condensa hitos en la historia de los sujetos, simbolizando en las marcas su propia versión, su propio relato. Este proceso de marcación, al igual que la memoria, jerarquiza e inscribe selectivamente en el cuerpo.

Y traemos una vez más al rostro, tema central, ya que si por un lado éste es una de las partes del cuerpo que muchas personar más resguardan de marcas y someten privilegiadamente a intervenciones en pos de alisar la piel para rejuvenecerlo (¿rejuvenecerlo?), por su parte intervenir este mismo espacio por medio del tatuaje 
requiere de una profunda seguridad; de hecho son tatuadores la mayoría de los que marcan su rostro, posiblemente por ser una de las zonas mas radicales dada su inevitable exposición. ${ }^{4}$ A propósito, lejos de perder su valor cardinal, en la sociedad espectacular el rostro es central. Junto con el corrimiento de los límites de mostración de la intimidad, cuya frontera en oposición a lo privado ha sido consentida y sensiblemente desplazada por el avance de la "extimidad" ${ }^{5}$ nuevos espacios se abren; tal es el caso de la Web en la que, a partir de variados dispositivos tecnológicos se habilitó la exposición generalizada (en algunos casos, minuto a minuto y en tiempo real) de la vida privada en el espacio público sin fronteras -no ya el de la plaza pública sino el del mundo entero, pero desde el resguardo del cuerpo en la comodidad y "seguridad" del espacio privado, del hogar-. Los espacios de comunicación virtual como fotologs, blogs, Facebook, Twitter, entre otros- estimulan la asociación a las cuentas de usuario, de una "foto de perfil". Ese ícono elegido por el sujeto para identificarse e individualizarse, en la mayoría de los casos coincide con una imagen del rostro del sujeto. Vemos que también la virtualidad en general recurre a un rostro (real o no) como elemento de identificación, aunque como bien apunta Sibilia, prescinde de la organicidad corporal, como de la materialidad del espacio y de la linealidad del tiempo (2005: 65).

Asumimos que el cuerpo y su imagen se construyen en la experiencia social, y que el tatuaje permitiría recuperarla en su forma analógica, como con-tacto con la realidad material próxima del mundo del sujeto. ¿Acaso estamos requiriendo del tatuaje más sentidos, respuestas, experiencias- de lo que su práctica concibe dar? Es posible.

\subsection{El cuerpo híbrido: ¿tatuaje como “bi(o)sutería"?}

Adornar el cuerpo es una de las prácticas humanas más antiguas, que en cada época adquirió su singularidad. Lo que se nombra actualmente como bi(o)sutería (derivación de la palabra francesa bijouterie) es la fusión del tradicional adorno del cuerpo en vinculación orgánica con lo virtual. Nos referimos por ejemplo a la transformación ideada por el australino artista-performer y profesor de arte, Sterlac quien convirtió "un pedazo de su propio cuerpo (en este caso un cartílago de su caja torácica) en una estética info-funcional (antena Internet)" (Andrieu; 2007: 27); de esto resultaría un cuerpo biovirtual, adaptado a los tiempos que corren, bajo el concepto de Sterlac de que el cuerpo es un caparazón obsoleto.

Como pequeños escalones en esta escalada de intervención técnica de los cuerpos, la empresa de tecnología, Nokia habría presentado este año en Estados Unidos de América una patente relacionada a los tatuajes-vibrantes que se aplicarían utilizando tintas ferromagnéticas para tatuar. Estos permitirían a los sujetos sincronizar los teléfonos móviles del tipo smartphones con el tatuaje para sentir los llamados o la

\footnotetext{
4 Tal es la relevancia del rostro que por ejemplo el médico y criminalista italiano Cesare Lombroso, redactó El hombre delincuente (1876), un manual en el que identificaba a partir de características, entre ellas del rostro y la cabeza, la fisiognomía del potencial delincuente.

${ }^{5}$ El concepto de "extimidad" es trabajado por Paula Sibilia en La intimidad como espectáculo (2008).
} 
llegada de mensajes. La alternativa también estaría contemplada con unos parches (como pequeños stickers) que podrían adherirse a la piel, y ser removidos a gusto, idea que engarza a la perfección con el ideal del mercado. La multiplicación de este tipo de gadgets, como pequeños accesorios tecnológicos, continúa restando espesura a la materialidad del cuerpo biológico, para construirlo cada vez como accesorio del sujeto constreñido por la ubicuidad de las tecnologías que decretan a la conexión como imperativo. Cuando todo es comunicación ya nada comunica; se reduce a contacto técnico, transmisión de información. Eso no es comunicación humana.

\section{CONCLUSIONES}

Sobre el final de la reflexión que propusimos resulta aun más nítida la imposibilidad de acercarnos a una conclusión, a una verdad, y francamente no esperamos eso. Alternativamente, esperamos poner en evidencia a partir de los elementos en juego en este trabajo, algunas piezas provisorias de un sistema que encontró un curso fecundo en nuestras sociedades y que viene presentándose como irrefrenable.

En el libro Arte y técnica (1952), Lewis Mumford advierte que "nuestras facilidades reproductivas en el campo de las artes tendrán valor humano sólo cuando aprendamos a poner freno a la inundación de imágenes y sonidos que ahora nos abruma, sólo cuando aprendamos a regular la ocasión, la cantidad, la duración, la frecuencia, conforme a nuestras necesidades, a nuestra capacidad de asimilación" (Mumford; 1952: 85. Cursiva en el original). De eso se trata tal vez, de restituirle valor humano, grosor, al sujeto, de re-centrar a/en la persona. Más de medio siglo nos separa del escrito de Mumford, sin embargo el mundo sigue girando sostenido en la misma embriaguez del exceso abrumador, de la saturación hastiosa. Con un plus de dramatismo se actualizan los temores del filósofo estadounidense, en la era de la tecnociencia, de lo postorgánico, parece cada vez menos posible volver al hombre sobre sus necesidades porque la materialidad de sí ya no le pertenece -o al menos no totalmente-. Qué es lo propio y qué lo ajeno.

Queremos insistir por último con Papalini en la posibilidad/necesidad de intensificar la experiencia subjetiva, enriqueciéndola para restituir poco a poco el grosor que le permita al sujeto aprovechar las posibilidades que genera el mecanismo de modulaciones en las sociedades contemporáneas, de las cuales el sujeto se apropiaría voluntariamente (2007: 42). Resuena con fuerza la propuesta de experimentar, de imaginar más allá de los márgenes establecidos para fortalecer las identidades que hagan posible incorporar las estructuras pero con mediaciones y mayores resistencias. Esta idea de la física de refracción todavía puede ser alimentada si se logran desarrollar $\mathrm{y}$ expandir identidades fuertes y subjetividades menos porosas que no teman identificar y poner en evidencia los síntomas de la época. Si sucede que las tecnologías están quitando grosor al lenguaje humano y convirtiendo al cuerpo en un objeto univoco, sin pliegues ni rugosidades, tal vez estas prácticas como el tatuaje vengan a ayudarnos a restituir por medio del cuerpo, la pluralidad de sentidos y experiencias que devuelvan al mundo lo múltiple. Necesitamos, como dice Weizenbaum recuperar la autonomía respecto de las máquinas, volver a acercar a los sujetos a la experiencia 
perdida a manos de la ciencia moderna. Aceptar como posibles el fracaso, lo no bello, el sufrimiento tal vez pueda comenzar a reconstruirnos en nuestra espesura humana, sabiendo que aquello que nos hace humanos, muchas veces, no tiene utilidad ni sentido.

\section{REFERENCIAS}

Andrieu, Bernard (2007) “Contra la desencarnación técnica: ¿un cuerpo híbrido?”. En Jean-Marc Lachaud y Olivier Neveux (directores), Cuerpos dominados, cuerpos en ruptura. Buenos Aires: Nueva Visión.

Butler, Judith (2002 [1993]) Cuerpos que importan: sobre los limites materiales y discursivos del "sexo". Buenos Aires: Paidós.

Ganter, Rodrigo (2006) "De cuerpos, tatuajes y culturas juveniles". En Revista Espacio Abierto, Asociación Venezonala de Sociología, Maracaibo: pp. 427-453.

Geertz, Clifford (1995) "El impacto del concepto de cultura en el concepto de hombre". En La interpretación de las culturas (pp. 43-59). Buenos Aires: Gedisa.

INDEC (2010) Censo Nacional de Población, Hogares y Viviendas 2010: total del país, resultados provisionales. Buenos Aires: Instituto Nacional de Estadística y Censos (INDEC).

Le Breton, David (2010) Rostros. Ensayo antropológico. Buenos Aires: Letra Viva. (2002) La sociología del cuerpo. Buenos Aires: Nueva Visión.

(2002a) Antropología del cuerpo y modernidad. Buenos Aires: Nueva

Visión.

Mumford, Lewis (1961 [1952]) Arte y técnica. Buenos Aires: Nueva Visión.

Papalini, Vanina (2007, enero-abril) “La domesticación de los cuerpos”. En Revista Enlace, año 4, volumen 1, Universidad de Zulia, Venezuela: pp. 39-53.

Reisfeld, Silvia (2005) "El cuerpo un enfoque interdisciplinario", en Tatuajes, una mirada psicoanalítica. Buenos Aires: Paidós.

Remondino, Georgina y Chomnalez, Valeria (2009, septiembre) “El docu-reality "Mundo Privado" ¿Un catalejo hacia esos "ocultos territorios juveniles"?, en Revista Oficios Terrestres, vol. 24. La Plata: pp. 99-109.

Sibilia, Paula (2005) El hombre postorgánico: Cuerpo, subjetividad y tecnologías digítales. Buenos Aires: Fondo de Cultura Económica. 
Schmucler, Héctor (1997) "El regreso de las palabras o los límites de la utopía mediática", en Héctor Schmucler, Memoria de la comunicación. Buenos Aires: Editorial Biblos.

Weizenbaum, Joseph (1978) "Los instrumentos", en La frontera entre el ordenador y la mente. Madrid: Ediciones Pirámide.

\section{Valeria Chomnalez}

Licenciada en Comunicación Social por la Universidad Nacional del Comahue, Argentina; becaria del Consejo Nacional de Investigaciones Científicas y Técnicas (CONICET), doctoranda en Estudios Sociales de América Latina del Centro de Estudios Avanzados (CEA) orientación Comunicación y Cultura de la Universidad Nacional de Córdoba, donde también cursa la Especialización en Investigación de la Comunicación; está realizando su tesis sobre jóvenes tatuadores. 